\title{
FLORA BRIOFÍTICA DA RESERVA BIOLÓGICA DO ALTO DA SERRA DE PARANAPIACABA, SÃO PAULO: 1 - LEJEUNEACEAE (HEPATICOPSIDA)(1)
}

\author{
Cristina Giancotti (2) \\ Daniel Moreira Vital (3)
}

\begin{abstract}
RESUMO - No levantamento de Lejeuneaceae (Hepaticopsida), na Reserva Biológica do Alto da Serra de Paranapiacaba, São Paulo, a partir de 1982, foi constatada a ocorrência de 14 gêneros e 20 espécies. Em contrapartida, pelo exame feito na coleção do herbário do Estado Maria Eneyda P. Kauffmann Fidalgo do Instituto de Botânica, da Secretaria do Meio Ambiente (SP) e por citações bibliográficas, foi verificada a existência de 25 gêneros e 66 espécies entre as décadas de 1920-1960. A diminuição do número de espécies consideradas sensíveis, provavelmente deve-se à poluição emanada do Polo Industrial de Cubatão.
\end{abstract}

Palavras-chaves: Hepaticopsida, Lejeuneaceae, Paranapiacaba/SP.

\begin{abstract}
During the survey of the Lejeuneaceae (Hepaticopsida) of the Alto da Serra de Paranapiacaba Biological Reserve, State of São Paulo, carried out from 1962 up today, 14 genera and 20 species were recorded. However, the checking of all herbarium materials of Instituto de Botânica (SP) and bibliographical references available led authors to the compilation of 25 genera and 66 species that were referred during the years 1920 to 1960 . Such a significant diminishing in the number of taxa, mostly represented by very sensitive species, is much probably due to the air pollution from Cubatão Industrial Complex.
\end{abstract}

Key words: Hepaticopsida, Lejeuneaceae, Paranapiacaba/SP.

\section{Introdução}

A ação do homem sobre a biosfera levou à diminuição ou mesmo a destruição total de numerosas espécies animais e vegetais, resultado de uma longa evolução. Este empobrecimento dos ecossistemas pela diminuição do número

(1) Trabalho executado dentro do programa Linhas de Ação em Botânica do CNPq - Mata Atlântica.

(2) Estagiária da Seção de Briologia e Pteridologia, Bolsista CNPq processo no 820.871-88.5.

(3) Seção de Briologia e Pteridologia, Instituto de Botânica - Caixa Postal 4005 - 01051 - São Paulo SP - Brasil. 
de indivíduos ou das espécies compromete a sua estabilidade e provoca conseqüências diversas.

$\mathrm{O}$ interesse em proteger certas zonas naturais ainda relativamente intacta materializou-se na criação de Parques Nacionais e Reservas Naturais em numerosos países. Pode-se definir sumariamente uma Reserva Natural como uma zona destinada a proteger de maneira "absoluta" um meio, com sua fauna e sua flora. É proibido exercer nela qualquer atividade humana, sendo a entrada reservada a alguns raros cientistas (DAJOZ, 1983). Assim, como vemos em nosso país pouco tem sido feito para atingir os objetivos de verdadeiros Parques Nacionais, Reservas Naturais e Estações Ecológicas.

Este é o caso da Reserva Biológica do Alto da Serra de Paranapiacaba, com uma área de 336 hectares e altitude média de $800 \mathrm{~m}$, estando localizada a $40 \mathrm{~km}$ SE da Praça da Sé em São Paulo e a $16 \mathrm{~km} \mathrm{NE}$ do centro de CubatãoSP, no município de Santo André, São Paulo.

Descrita por HOEHNE (1925) como um local de rara beleza, e com uma vegetação de Mata Atlântica intacta, hoje sofre com os efeitos da poluição emanada do polo Industrial de Cubatão, devendo este ser o principal fator de um gradativo desaparecimento das espécies sensíveis.

No Alto da Serra são pouco caracterizadas as quatro estações do ano. A umidade é intensa, resultante de brisas marítimas e da temperatura elevada da massa atmosférica aquecida pela superfície oceânica (MATTOS FILHO, 1930). Esta massa atmosférica trazendo em suspensão as partículas emanadas deste Polo Industrial ao defrontar com a Serra sofre um brusco resfriamento e conseqüênte condensação, sob a forma de neblina, garoa ou chuva, depositando aí estas partículas.

Assim, o objetivo deste estudo é realizar um inventário da situação atual da flora da família Lejeuneaceae (Hepaicopsida) da Reserva Biologica do Alto da Serra de Paranapiacaba, na tentativa de avaliar as condiçöes ambientais presentes e a comparar com os dados obtidos em décadas passadas.

\section{Material e Métodos}

Para o estudo taxonômico foi utilizado:

- Materiais depositados no herbário do Estado Maria Eneyda P. Kauffmann Fidalgo, do Instituto de Botânica da Secretaria do Meio Ambiente, (SP);

- Materiais coletados nas áreas da Reserva Biológica do Alto da Serra de Paranapiacaba a partir de 1962;

- As identificações foram feitas pela morfologia comparada, utilizando-se a bibliografia especializada principalmente os trabalhos de: HERZOG (1925), SCHIFFNER \& ARNELL (1964), BISCHLER (1964) E SCHUSTER (1980).

\section{Resultados}

A família Lejeuneaceae é bem típica de áreas com vegetação higrófila, sendo que as condições de umidade, temperatura e luminosidade da Reserva 
Biológica, seriam excelente para a existência de uma exuberante e variada associação de gêneros e espécies desse grupo. No entanto as coletas realizadas mostram somente as seguintes espécies:

-Anoplolejeunea conferta (Meissn. ex Spreng.) Evans

Material examinado: 20-I-1966, Vital 556; 14-II-1978, Vital 7850, 7854.

- Cheilolejeunea acutangula (Ness) Grolle

Material examinado: 1-II-1961, A. Baranov 105.

- Colojeunea minutissima (Sm.) Schiffn.

Material examinado: 6-XII-1988, Giancotti 133.

- Colura tenuicornis (Evans) Steph.

Material examinado: 5-V-1982, Vital 10421; 6-XII-1988 Giancotti 137, 139; 17-III-1989, Giancotti 145.

- Crossotolejeunea crenata (Mont. \& Nees) Steph.

Material examinado: 28-X-1962, Eiten \& Eiten 5026

- Cyclolejeunea luteola (Spruce) Grolle.

Material examinado: 31-VIII-1988, De Vuono et al. 31; 3-I-1989, De Vuono et al. 439.

- Drepanolejeunea fragilis Bischler

Material examinado: 17-III-1962, Vital 86.

- Drepanolejeunea lichenicola (Spruce) Steph.

Material examinado: 20-I-1966, Vital 556-B.

- Drepanolejeunea mosenii (Steph.) Bischler

Material examinado: 14-II-1978, Vital 7850, 7856; 4-V-1982, Vital 10398; 5-V-1982, Vital 10406; 26-II-1985, Yano 9410; 13-VIII-1985, Vital 13140; 17-II-1987, Vital 14798; 8-XI-1988, Giancotti 132; 6-XII-1988, Giancotti 133,134 .

- Drepanolejeunea sabaliana Schuster

Material examinado: 14-II-1978, Vital 7852, 7853, 7854, 7855, 7857; 26XI-1980, Yano 3165; 4-V-1982, Vital 10400; 5-V-1982, Vital 10419; 26II-1985, Yano 9393, 9401, 9403, 9410; 7-VI-1988, Giancotti 103, 104, 105, 106, 107, 108, 110; 9-VIII-1988, Giancotti 119; 8-XI-1988, Giancotti 123.

- Harpalejeunea ancistrodes (Sprice) Steph.

Material examinado: 20-I-1966, Vital 556-A.

- Harpalejeunea oxyphylla (Nees \& Mont.) Steph.

Material examinado: 13-VIII-1985, Vital 13142.

- Lejeunea flava (sW.)Nees

Material examinado: 10-III-1964, Vital 179, 181; 14-II-1973, Vital 7856; 17 IV-A980, Custódio Filho 228; 4-V-1982, Vital 10394; 5-V-1982, Vital 10406, 10429; 13-VIII-1985, Vital 13140; 20-II-1985, Yano 9403, 9409; 19-VI-1986, Vital 13849; 17-II-1987, Vital 14798; 7-VI-1988, Giancontti 102,-103, 104, 106, 107, 109; 9-VIII-1988, Giancotti 113, 116; 8-XI-1988, Giancotti 130, 134.

- Lejeunea glaucescens Gott.

Material examinado: 24-IV-1987, Vital 14820.

- Lejeunea ulicina subsp. bullata (Tayl.) Schuster 
Material examinado:13-VIII-1985, Vital 13140.

- Leucolejeunea xanthocarpa (Lehm. \& Lindenb.) Evans

Material examinado: 4-V-1982, Vital 10401; 17-III-1989, Giancotti 146.

- Microlejeunea cystifera Herz.

Material examinado: 28-X-1962, Eiten \& Eiten 5026.

- Omphalanthus filiformis (Sw.) Nees

Material examinado: 28-X-1962, Eiten \& Eiten 5025; 13-VIII-1985, Vital 13142.

- Rectolejeunea emarginuliflora (Gott.) Evans

Material examinado: 8-IX-1988, De Vuono et al. 715.

- Taxilejeunea isocalycina (Nees) Steph.

Material examinado: 17-III-1962, Vital 91.

Pelas amostras do herbário do Estado Maria Eneyda P. Kauffmann Fidalgo, do Instituto de Botânica (SP), foram registradas espécies coletadas na década de vinte:

- Bryopteris diffusa (Sw.) Nees

Material examinado: 23-XII-A920, Hoehne s/n.

- Ceratolejeunea fallax (Lehm. \& Lindenb.) Bonner

Material examinado: 4-VII-1922, A. Gehrt 493.

- Cheilolejeunea acutangula (Nees) Grolle

Material examinado: 23-XII-1920, Hoehne 145.

- Cheilolejeunea trifaria (Reinw. et al.) Mizut.

Material examinado: IX-1986, Loefgren 756.

- Cyclolejeunea convexistipula (Lehm. \& Lindenb.) Evans

Material examinado: 4-VII-1922, A. Gehrt 469, 493, 497, 499, 500, 520, 543.

- Cyclolejeunea papillata Steph.

Material examinado: 4-VII-1922, A Gehrt 495, 501, 502, 524, 542.

- Drepanolejeunea anoplantha (Spruce) Steph.

Material examinado: 3-VIII-1921, A. Gehrt 340.

- Drepanolejeunea mosenii (Steph.) Bischler

Material examinado: 3-VIII-1921, Hoehne 342; 4-VII-1922, A. Gehrt 492; I-1923, Hoehne 544.

- Drepanolejeunea orthophylla (Nees \& Mont.) Bischler

Material examinado: 3-VIII-1921, Hoechne 342; 4-VII-1922, A. Gehrt 492;

I-1923, Hoehne 544.

- Leucolejeunea xanthocarpa (Lehm. \& Lindenb.) Evans

Material examinado: 23-II-1920, A. Gehrt 105.

- Odontolejeunea angustifolia Steph.

Material examinado: 3-VIII-1921, A. Gehrt 339; 4-VII-1922, A. Gehrt 495.

- Odontolejeunea fissistipula Steph.

Material examinado: 3-VIII-1921, Hoehne 340.

- Odontolejeunea lunulata (Web.) Schiffn.

Material examinado: 6-X-1922, A. Gehrt 533; 6-X-1922, A. Gehrt 526, 527. 
Flora briofítica da Reserva Biológica...

- Omphalanthus filiformis (Sw.) Nees

Material examinado: 23-XII-1920, Hoehne 136.

- Strepsilejeunea apicidens Herz.

Material examinado: 21-IV-1920, Herzog s/n.

Através das citações bibliográficas foram encontradas:

- Anoplolejeunea conferta (Meissn. ex Spreng.) Evans

HERZOG (1927).

- Aphanolejeunea exigua Evans

SCHIFFNER \& ARNELL (1964).

- Aphanolejeunea kunertiana Steph.

SCHIFFNER \& ARNELL (1964).

- Aphanolejeunea liliputiana (Spruce) Schuster

SCHIFFNER \& ARNELL (1964) como Cololejeunea liliputiana (Spruce) Arnell.

- Aphanolejeunea sicaefolia (Gott. in Steph.) Evans

SCHIFFNER \& ARNELL (1964).

- Bryopteris filicina (Sw.) Nees

STOTLER \& CRANDALL (1974).

- Ceratolejeunea fallax (Lehm. \& Lindenb.) Bonner

HERZOG (1927) como Ceratolejeunea brasiliensis (Gott.) Steph.

- Ceratolejeunea longicornis (Gott.) Steph.

SCHIFFNER \& ARNELL (1964).

- Ceratolejeunea rufo-pellucida (Spruce) Steph.

SCHIFFNER \& ARNELL (1964).

- Cheiloleieunea comans (Spruce) Schuster

GROLLE (1988)

- Cololejeunea ensifolia (Spruce) Steph.

SCHIFFNER \& ARNELL (1964).

- Cololejeunea mosenii (Steph.) Arnell

SCHIFFNER \& ARNELL (1964).

- Cololejeunea platyneura (Spruce) Arnell

SCHIFFNER \& ARNELL (1964).

- Colura tortifolia (Mont. ex Nees) Trev.

SCHIFFNER \& ARNELL (1964).

- Cyclolejeunea grandistipula Steph.

SCHIFFNER \& ARNELL (1964).

- Cyclolejeunea convexistipa (Lehm. \& Lindenb.) Evans

HERZOG (1927).

SCHIFFNER \& ARNELL (1964) como Cyclolejeunea grossidens Steph.

- Cyclolejeunea papillata Steph.

HERZOG (1927).

- Cyclolejeunea paulina (Steph.) Steph.

SCHIFFNER \& ARNELL (1964). 
- Cyclolejeunea peruviana (Lehm. \& Lindenb.) Evans

HERZOG (1927)

SCHIFFNER \& ARNELL (1964).

- Cystolejeunea lineata (Lehm. \& Lindenb.) Evans

SCHIFFNER \& ARNELL (1964).

- Dicranolejeunea aberrans (Lindenb. \& Gott.) Steph.

SCHIFFNER \& ARNELL (1964) como Dicranolejeunea paulina Gott. ex Steph..

- Diplasiolejeunea brunnea Steph.

SCHIFFNER \& ARNELL (1964).

- Diplasiolejeunea cavifolia Steph.

REYES (1982).

Diplasiolejeunea pellucida (Meissn.) Schiffn.

SCHIFFNER \& ARNELL (1964).

- Drepanolejeunea aculeata Bischler

BISCHLER (1964).

- Drepanolejeunea anoplantha (Spruce) Steph.

HERZOG (1925 e 1927) como Drepanolejeunea subulata Steph.

- Drepanolejeunea araucariae Steph. var. araucariae

BISCHLER (1964).

- Drepanolejeunea biocellata Evans

HERZOG (1927).

SCHIFFNER \& ARNELL (1964).

- Drepanolejeunea campanulata (Spruce) Steph.

SCHIFFNER \& ARNELL (1964).

- Drepanolejeunea capulata (Tayl.) Steph.

SCHIFFNER \& ARNELL (1964).

- Drepanolejeunea mosenii (Steph.) Bischler

SCHIFFNER \& ARNELL (1964) como Drepanolejeunea lancifolia (Gott.)

Steph.

BISCHLER (1967).

- Drepanolejeunea orthophylla (Nees \& Mont.) Bischler

SCHIFFNER \& ARNELL (1964) como Leptolejeunea ungüiculata Steph., Leptolejeunea hamulata (Gott.) Schiffn. ex Steph. e Leptolejeunea stenophylla (Lindenb. \& Gott.) Steph.,

HERZOG (1925, 1927) como Leptolejeunea stenophylla (Lindenb. \& Gott.) Steph.

- Drepanolejeunea subdissitifolia Herz.

HERZOG (1949).

BISCHLER (1964).

- Euosmolejeunea beyrichii (Lindenb.) Steph.

HERZOG (1927).

SCHIFFNER \& ARNELL (1964).

- Harpalejeunea lignicola (Spruce) Steph.

SCHIFFNER \& ARNELL (1964). 
- Harpalejeunea oxyphylla (Mont. et Nees) Steph.

HERZOG (1927).

- Harpalejeunea verrucosa Herz.

HERZOG (1949).

- Lejeunea flava (Sw.) Nees

SCHIFFNER \& ARNELL (1964).

- Lejeunea monimiae (Steph.) Steph.

SCHIFFNER \& ARNELL (1964).

- Lejeunea pterogonia (Lehm. \& Lindenb.) Mont.

SCHIFFNER \& ARNELL (1964) como Taxilejeunea pterogonia (Lehm. \& Lindenb.) Schiffn.

- Lejeunea ulicina subsp. bullata (Tayl.) Schuster

SCHIFFNER \& ARNELL (1964) como Microlejeunea bullata (Tayl.)

- Leptolejeunea elliptica (Lehm. \& Lindenb.) Steph.

SCHIFFNER \& ARNELL (1964).

BISCHLER (1969).

- Leucolejeunea unciloba (Lindenb.) Evans

SCHIFFNER \& ARNELL (1964) como Leucolejeunea sellowiana Steph..

- Leucolejeunea xanthocarpa (Lehm. \& Lindenb.) Evans

HERZOG (1925) como Archilejeunea involuta Steph.,

HERZOG (1927).

- Microlejeunea aphanes (Spruce) Steph.

SCHIFFNER \& ARNELL (1964).

- Microlejeunea corcovadae (Gott. ex Steph.) Steph.

SCHIFFNER \& ARNELL (1964).

- Microlejeunea cystifera Herz.

HERZOG (1949),

SCHIFFNER \& ARNELL (1964).

- Microlejeunea subaphanes Herz.

HERZOG (1949),

SCHIFFNER \& ARNELL (1964).

- Microlejeunea subulistipa Steph.

SCHIFFNER \& ARNELL (1964).

- Odontolejeunea angustifolia Steph.

HERZOG (1927).

- Odontolejeunea lunulata (Web.) Schiffn.

HERZOG (1927),

SCHIFFNER \& ARNELL (1964).

- Odontolejeunea sieberiana (Gott.) Schiffn. var. spinosa Arnell

SCHIFFNER \& ARNELL (1964).

- Omphalanthus filiformis (Sw.) Nees

HERZOG (1925).

- Omphalanthus grandistipulus Steph.

SCHIFFNER \& ARNELL (1964).

- Otigolejeunea apiahyana Steph. 
SCHIFFNER \& ARNELL (1964).

- Prionolejeunea fendleri Steph.

HERZOG (1927).

- Prionolejeunea limpida Herz.

HERZOG (1927) como Prionolejeunea submegalantha Herz., SCHIFFNER \& ARNELL (1964) como Prionolejeunea scaberula

(Spruce) Steph..

- Prionolejeunea prionodes Steph.

SCHIFFNER \& ARNELL (1964).

- Rectolejeunea mandiocana Steph.

SCHIFFNER \& ARNELL (1964).

- Taxilejeunea iheringii Steph.

SCHIFFNER \& ARNELL (1964).

\section{Conclusāo}

Estes resultados mostram que provavelmente o gradativo desaparecimento de espécies consideradas sensíveis deve-se a poluição, e assim as poucas espécies que se mostraram tolerantes aumentaram muito a sua quantidade ocupando os nichos ecológicos deixados pelas espécies sensíveis.

Convém salientar ainda que no passado as coletas eram feitas esporadicamente, o que leva a supor que deveria haver na região muito mais espécies do que as conhecidas.

\section{Referências Bibliográficas}

BISCHLER, H. 1964. Le genre Drepanolejeunea Steph. em Amérique Centrale et Méridionale. Rev. bryol. lichen., 33 (1-2): 15-179.

—. 1967. Le genre Drepanolejeunea Steph. in Amérique Centrale et Méridonale. -II. Rev. bryol. lichen., 35 (1-4): 95-134.

—. 1969. Le genre Leptolejeunea (Spruce) Steph. em Amérique. Nova Hedwigia, 17: 265-350.

DAJOZ, R. 1983. Ecologia Geral. Editora da USP, São Paulo. 472 p.

GROLLE, R. 1988. Miscellanea Hepaticologica 261-270. J. Hattori bot. Lab., 65: 403-410.

HERZOG, T. 1925. Contribuição ao conhecimento da flora bryológica do Brasil. Arch. Bot. S. Paulo, 1 (2): 27-105.

—. 1927. Zwei Bryophyten sammlungen aus Südamerika. Hedwigia, 67: 249-268.

—. 1949. Miscellanea Bryologica I. Neotropica. Memor. Soc. Fauna Flor. fenn., 25: 43-73.

HOEHNE, F. C. 1925. A Estação do Alto da Serra. Album da Seção de Botânica do Museu Paulista, 215p.

MATTOS FILHO, B. 1930. A Estação Biológica do Alto da Serra sob o prisma meteorológico. Arch. Inst. biol. (Def. Agric. anim,), São Paulo, 3: 109-127.

REYS, D. M. 1982. El genero Diplasiolejeunea en Cuba. Acta Bot. Acad. Sci. Hungaricae, 28 (1-2): 145-180.

SCHIFFNER, V. \& S. ARNELL. 1964. Ergebnisse der Botanischen Expedition der Kai- 
Flora briofítica da Reserva Biológica...

serlichen Akademie der Wissenchaften Nach Südbrasilien 1901. II. Band (Thallophyta und Bryophyta). Hepaticae. Osterr. Akad. Wiss. Math. -Nat. K., Denkschr., 111: 1-156.

SCHUSTER, R. M. 1980. The Hepaticae and Anthocerotae of North America east of hundredth Meridian. Columbia University Press. pp. 706-1314.

STOTLER, R. \& B. CRANDALL-STOTLER. 1974. A monograph of the genus Bryopteris (Swartz) Ness von Esenbeck. Bryophytorum Biblthca, 3: 1-159. 